\title{
Follicular regulatory $T$ cells inhibit the development of granzyme B-expressing follicular helper T cells
}

\author{
Markus M. Xie,, ${ }^{1}$ Shuyi Fang, ${ }^{2}$ Qiang Chen, ${ }^{1}$ Hong Liu, ${ }^{1}$ Jun Wan, ${ }^{2,3,4}$ and Alexander L. Dent ${ }^{1}$ \\ 1Department of Microbiology and Immunology, Indiana University School of Medicine, Indianapolis, Indiana, USA \\ ${ }^{2}$ Indiana University School of Informatics and Computing, Indiana University-Purdue University Indianapolis, Indianapolis, \\ Indiana, USA. ${ }^{3}$ Department of Medical and Molecular Genetics and ${ }^{4}$ Center for Computational Biology and Bioinformatics, \\ Indiana University School of Medicine, Indianapolis, Indiana, USA.
}

T follicular regulatory (TFR) cells are found in the germinal center (GC) response and help shape the antibody (Ab) response. However, the precise role of TFR cells in the CC is controversial. Here, we addressed TFR cell function using mice with impaired TFR cell development (Bcl6-flox/Foxp3-cre, or Bcl6FC mice), mice with augmented TFR cell development (Blimp1-flox/Foxp3-cre, or Blimp1FC mice), and two different methods of immunization. Unexpectedly, GC B cell levels positively correlated with TFR cell levels. Using a gene profiling approach, we found that TFH cells from TFRdeficient mice showed strong upregulation of granzyme B $(\mathrm{Gzmb})$ and other effector CD8+ ${ }^{+}$cell genes, many of which were Stat4 dependent. The upregulation of cytotoxic genes was the highest in TFH cells from TFR-deficient mice where Blimp1 was also deleted in Foxp3+ regulatory T cells (Bcl6-flox/Prdm1-flox/Foxp3-cre [DKO] mice). Granzyme B- and Eomesodermin-expressing TFH cells correlated with a higher rate of apoptotic GC B cells. KIrg1+ TFH cells from DKO mice expressed higher levels of Gzmb. Our data show that TFR cells repress the development of abnormal cytotoxic TFH cells, and the presence of cytotoxic TFH cells correlates with a lower GC and Ab response. Our data show what we believe is a novel mechanism of action for TFR cells helping the GC response.

Conflict of interest: The authors have declared that no conflict of interest exists.

Copyright: (c) 2019, American Society for Clinical Investigation.

Submitted: February 11, 2019

Accepted: July 16, 2019

Published: August 22, 2019.

Reference information: JCI Insight. 2019;4(16):e128076. https://doi.org/10.1172/jici. insight.128076.

\section{Introduction}

Abs with high affinity for antigen (Ag) are produced by B cells that are selected in the germinal center (GC) reaction (1-3). T follicular helper (TFH) cells are critical for this selection process (1-3). TFH cells provide survival and proliferative signals to GC B cells via CD40 ligand as well as the secretion of key cytokines such as IL-4 and IL-10 (1-3). Ab responses are also modulated by T follicular regulatory (TFR) cells, a subset of Foxp3 $3^{+}$regulatory T cells that express CXCR5 and localize to the GC (4-6). Both TFR cells and TFH cells are dependent on the transcriptional repressor protein Bcl6 for their development (3-7). TFR cells are derived from Foxp $3^{+}$regulatory $\mathrm{T}$ (Treg) cells and have been reported to repress excessive TFH cell and GC B cell proliferation as well as promote the selection of high-affinity B cells (8-11). The suppressor mechanisms used by TFR cells to suppress the GC are not well defined. Furthermore, a few recent studies have challenged the suppressor model of TFR cell function (reviewed in ref. 9).

A mouse model in which the Bcl6 gene is specifically deleted in Foxp3 ${ }^{+} \mathrm{T}$ cells (Bcl6-flox Foxp3-Cre, or Bcl6FC mice), blocking TFR cell development, has been used to precisely target TFR cell development in vivo (7, 12-14). This Bcl6FC mouse model has helped to show that TFR cells can help promote the Ag-specific IgG and GC response, in part by expressing the cytokine IL-10, which can promote GC B cell growth $(7,12)$. However, the full functions of TFR cells in the GC remain unclear and particularly it is unknown if TFR cells use other mechanisms besides IL-10 to help augment GC B cell responses and Ab production. Here we examined TFH cell gene expression in TFR cell-deficient and TFR cell-amplified mouse strains and we find that TFR cells are critical for inhibiting the development of an aberrant TFH cell subset with cytotoxic potential. Our data show that TFR cells control TFH gene expression and point to a mechanism for how TFR cells can promote the GC and $\mathrm{Ab}$ response. Our findings also raise the intriguing prospect that TFH cells can control the GC and Ab response by a cytotoxic mechanism. 


\section{Results}

TFR cells promote stronger GC B cell and IgE responses. To study Ag-specific Ab responses, we used a classic model of food allergy induced by challenge intragastrically (i.g.) with peanut protein plus cholera toxin (PCT) (15). In this model, high levels of peanut-specific IgE are induced and maintained in circulation for weeks after immunization in normal mice. To test the role of TFR cells, we used 3 conditional knockout strains with altered TFR cell development: "Bcl6FC" mice with Bcl6 deletion in Foxp3 ${ }^{+}$cells and so lack TFR cells (7), "Blimp1FC" mice with Prdm1 (Blimp1) deletion in Foxp3 $3^{+}$cells leading to augmented TFR cells (16), and "DKO" mice with deletion of both Bcl6 and Prdm1 in Foxp3 $3^{+}$cells. All of these mouse strains were grossly healthy with no obvious disease up to 10 weeks of age. As shown in Figure 1A, TFR cells were largely ablated in Bcl6FC and DKO mice but were increased about 2-fold over WT levels in Blimp1FC mice, similar to previously reported observations (16). The lack of TFR cells in DKO mice shows that Bc16 has a dominant role in promoting TFR cell development over repression by Blimp1. Loss of TFR cells did not affect TFH cell numbers in Bcl6FC mice; however, there was a marked increase in both Blimp1FC and DKO TFH cells (Figure 1B). Blimp1 is required for IL-10 expression by Tregs (17), indicating that Tregs from both Blimp1FC and DKO mice are defective in IL-10 expression. Therefore, the data in Figure 1B suggest that, more than TFR cells, Treg-derived IL-10 controls the expansion of TFH cells. In contrast, GC $B$ cell numbers showed a clear positive correlation with TFR cells in the 4 mouse strains (Figure 1C). There was a 5- to 6-fold lower ratio of GC B cells to TFH cells in TFR-deficient mice compared with TFR-sufficient mice, indicating that TFR cells increase the helper function of TFH cells in the GC (Figure 1, D and $\mathrm{E})$. Analysis of peanut-specific $\mathrm{Ab}$ titers revealed that TFR cells were also required for sustained and robust peanut-specific IgE and IgG1 responses in this model (Figure 1F). Overall, these findings support the idea that TFR cells act as helper cells in the GC and promote the Ag-specific IgE response.

TFR cells inhibit the development of aberrant cytotoxic gene-expressing TFH cells, particularly in the context of an inflammatory environment. To better understand how TFR cells were influencing the ability of TFH cells to help GC B cells, we used RNA sequencing (RNAseq) to profile gene expression in TFH cells from PCT-challenged WT, Bcl6FC, Blimp1FC, and DKO mice (Figure 2 and Supplemental Figure 2A; supplemental material available online with this article; https://doi.org/10.1172/jci.insight.128076DS1). TFH gene expression was strongly affected by loss of TFR cells, leading to several hundred up- and downregulated differentially expressed genes (DEGs) for both Bc16FC and DKO TFH cells (Figure 2A). Gzmb (granzyme B), a gene associated with cytotoxic T cells, stood out as being strongly elevated in TFH cells from both Bcl6FC and DKO mice, strains both lacking TFR cells (Figure 2A). Compared with WT TFH cells, Gzmb was increased 9-fold in Bcl6FC TFH cells and increased 66-fold in DKO TFH cells. Comparing DKO TFH cells to Blimp1FC TFH cells, Gzmb was increased 173-fold in DKO TFH cells. Despite the high number of DEGs, the expression of key TFH genes was essentially normal in Bcl6FC and DKO TFH cells (Figure 2B). To better understand the nature of the DEGs in Bcl6FC and DKO TFH cells, we compared upregulated DEGs from our data set with published gene expression data sets. We found a highly significant enrichment of genes that were at least 2-fold upregulated in effector CD8 ${ }^{+} \mathrm{T}$ cells (18) or more than 2-fold downregulated in Stat4 $4^{-1-}$ Th1 cells (19) within the upregulated DEGs from Bc16FC and DKO TFH cells (Figure 2C and Supplemental Figure 1). Next, we selected a set of 23 genes cells that were highly upregulated in Bcl6FC and $\mathrm{DKO} \mathrm{TFH}$ and also associated with $\mathrm{CD} 8^{+}$effector $\mathrm{T}$ cell differentiation (refs. 18, 20, and Figure 2D). About two-thirds (15 of 23) of these genes were both upregulated during $\mathrm{CD}^{+}$effector $\mathrm{T}$ cell differentiation (Figure $2 \mathrm{E}$ ) and also positively regulated by Stat 4 (Figure 2F). Unexpectedly, the expression of the $\mathrm{CD}^{+}$effector genes was nearly identical between WT and Blimp1FC TFH cells, even though Blimp1FC mice have defective Treg cell function and develop inflammatory disease $(21,22)$. $\mathrm{CD}^{+}$cytotoxic effector genes were upregulated to a higher degree in DKO TFH cells than in Bcl6FC TFH cells (Figure 2, C and D), indicating that loss of TFR cells together with loss of Blimp1 in Tregs in the DKO mice promoted a synergistic loss of control in TFH cell differentiation. Blimp1FC and DKO Tregs cannot produce IL-10 due to Blimp1 deficiency and DKO Tregs cannot develop into TFR cells due to Bcl6 deficiency. The deficiency of Treg-derived IL-10 could lead to an inflammatory environment in vivo that contributes to the greatly increased development of cytotoxic-like TFH cells in DKO mice. Additionally, despite significant overlap of the cytotoxic T cell gene program with Stat 4 function, the cytotoxic gene program appeared to be a unique non-Th1 differentiation state, as there were no significant increases in Ifng and Txb21 (Tbet) (Figure 3A), nor was there an enrichment of Tbet target genes in the cytotoxic TFH genes from Bcl6FC and DKO mice (Figure 3B). We then 
A

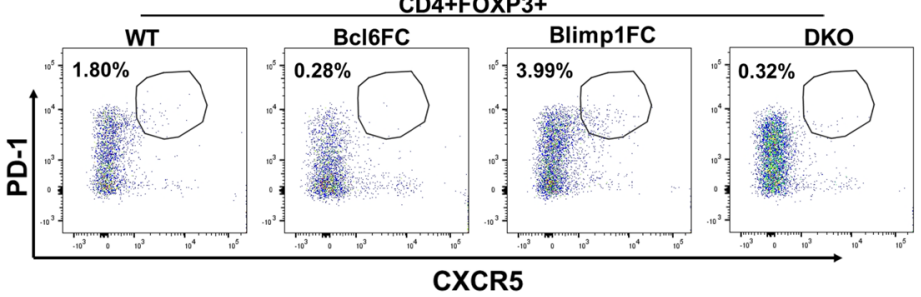

B

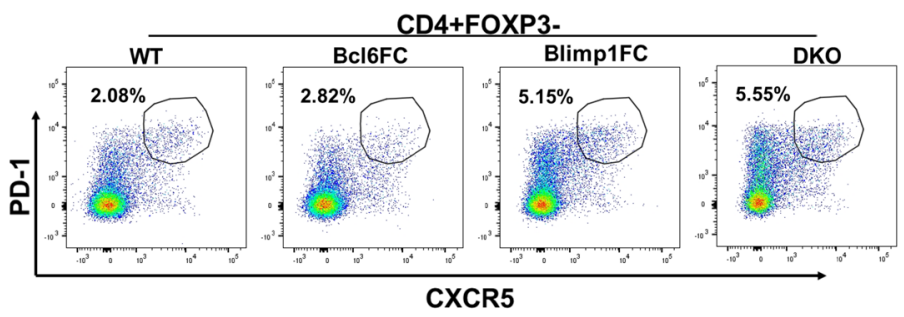

C

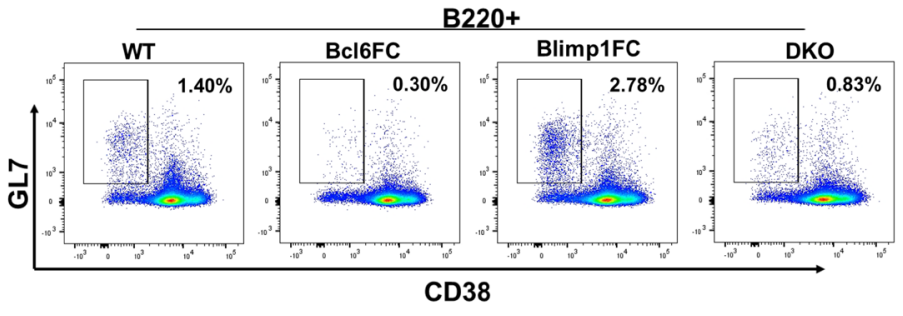

D

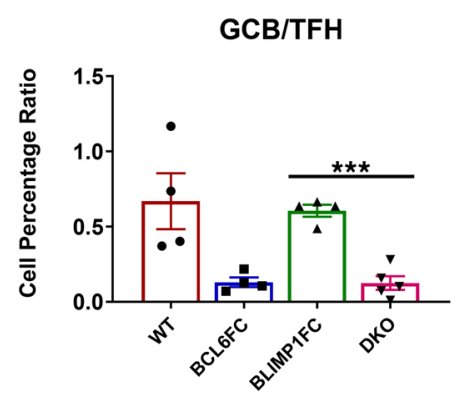

$\mathbf{F}$
Anti-Peanut Extract lgE

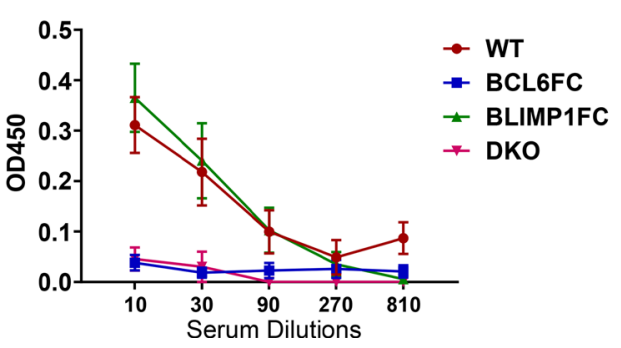

Anti-Peanut Extract IgG1

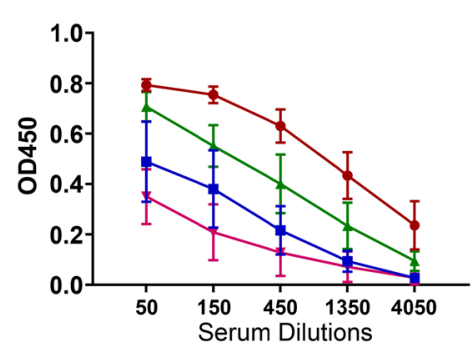

SP TFR\%

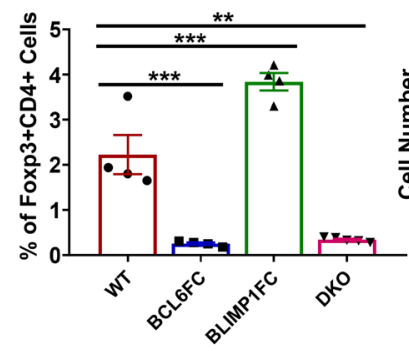

SP TFH\%
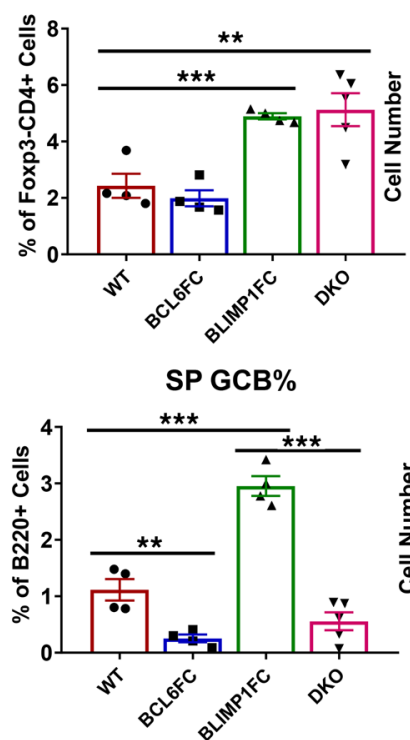

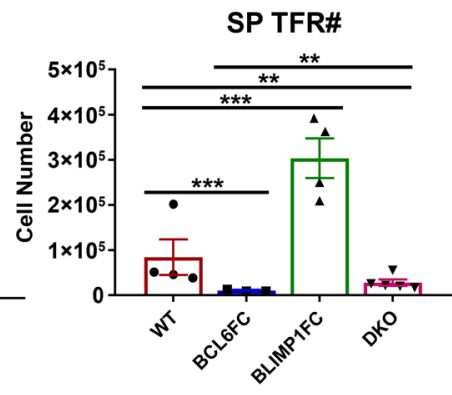

SP TFH\#
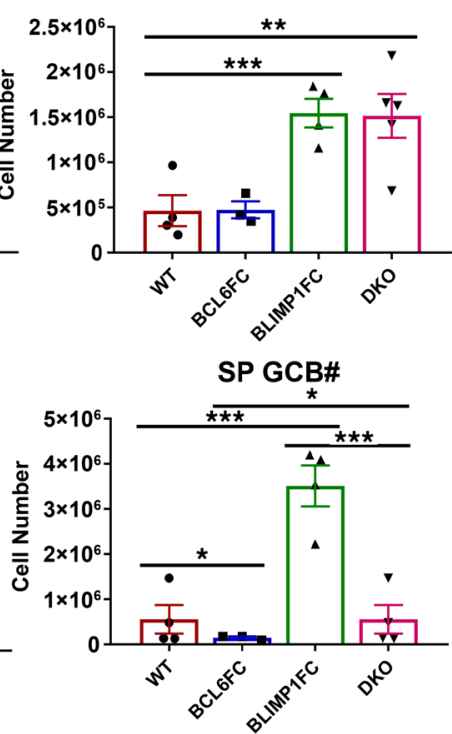

$\mathbf{E}$

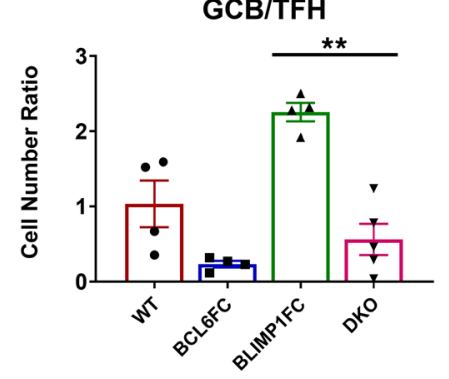

Figure 1. TFR cells are required for proper GC B cell numbers in a food allergy immune response. WT, BcI6FC, Blimp1FC, and DKO mice were orally immunized twice with peanut protein plus cholera toxin (PCT). Four weeks after the last PCT immunization (day 36), spleens (SP) were analyzed for the indicated cell populations by flow cytometry. Representative contour dot plots for each cell staining are shown along with graphs showing average percentage of cells as a fraction of parental cell population. (A) Analysis of CD4 ${ }^{+} \mathrm{FOX}$ P3+PD-1+CXCR5 ${ }^{+}$TFR cells. Average TFR cells per group are quantitated as a percentage (\%) of $\mathrm{FOXP3}^{+} \mathrm{CD}^{+}$ T cells, and absolute number (\#). (B) Analysis of CD4 ${ }^{+}$FOXP3-PD-1+CXCR5 ${ }^{+}$TFH cells. Average TFH cells are quantitated as a percentage of FOXP3-CD4+ $\mathrm{T}$ cells, and absolute number. (C) Analysis of B220 ${ }^{+} \mathrm{CD} 38^{-} \mathrm{CL} 7^{+}$ GC B cells. Average GC B cells per group graphed as a percentage of $\mathrm{B}^{2} 2 \mathrm{O}^{+}$cells and as absolute number. (D and $\mathbf{E}$ ) Ratio of GC B cells to TFH cells from data in $\mathbf{A}-\mathbf{C}$. (F) Titers of peanut-specific IgE and IgG1 by ELISA at day 36 . Graphs show the mean \pm SEM. $P$ values were calculated by $t$ test, where ${ }^{*} P<0.05$, ${ }^{* *} P<0.01$, ${ }^{* * *} P<$ 0.0001. $n=4-6$ mice. Data shown are representative of 3 experiments with similar results. ANOVA with Tukey's post hoc analysis was used to determine statistical significance. 
A - Up-DEG • Down-DEG • Non-DEG
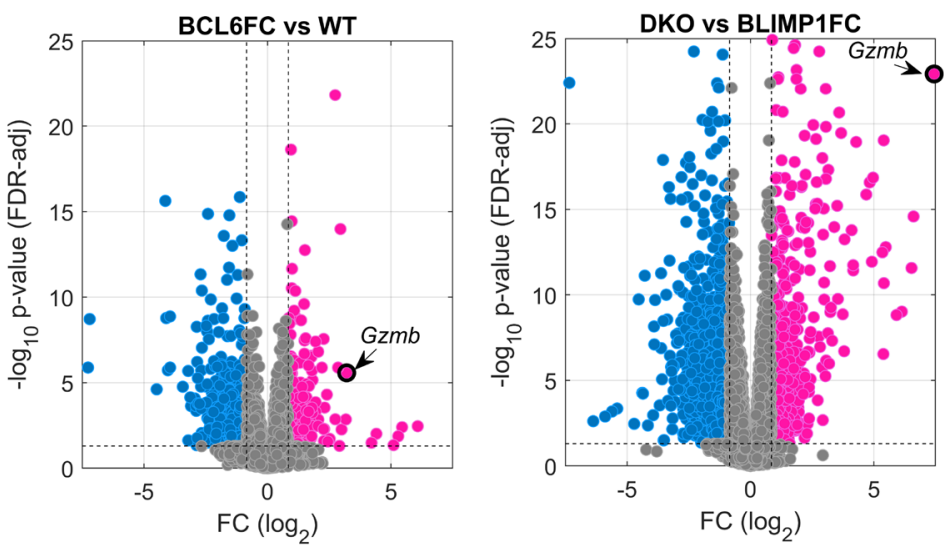

C
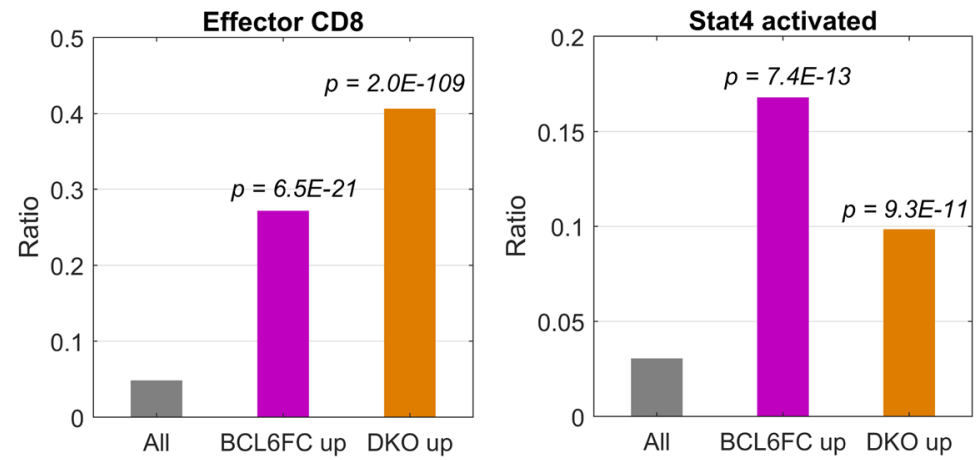

D
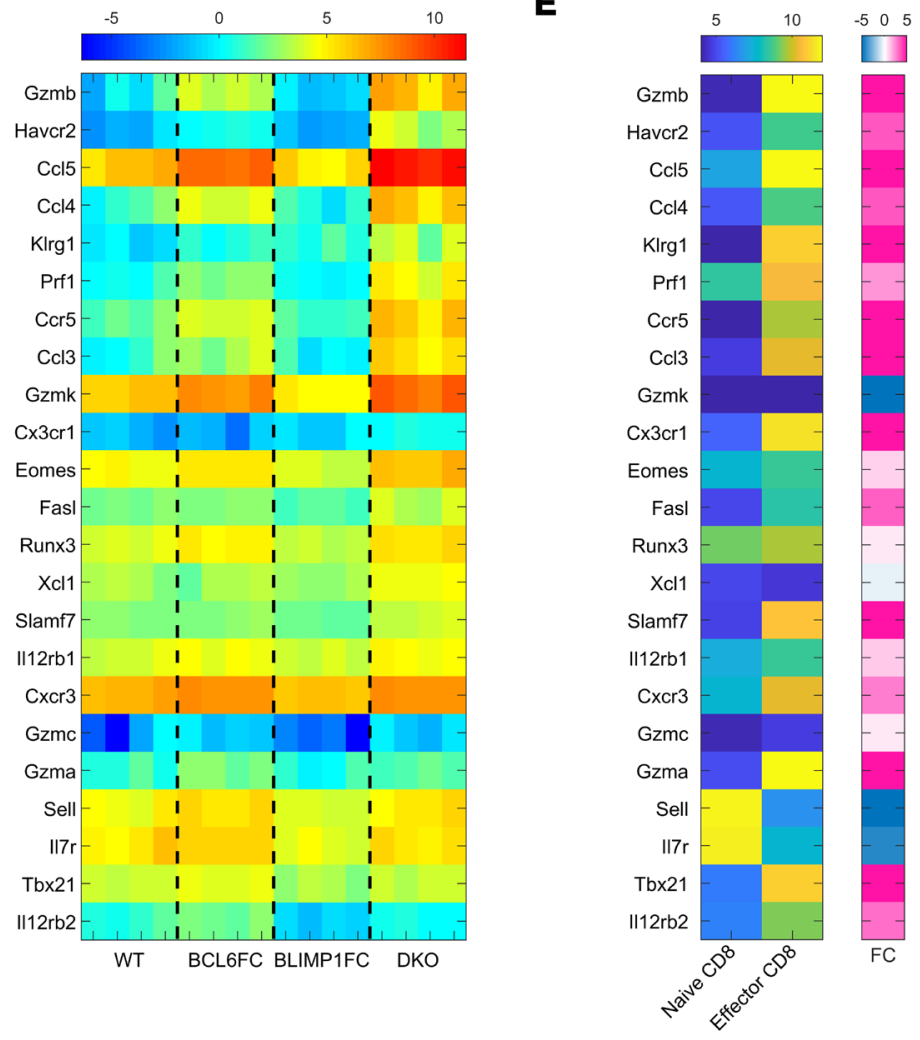

B

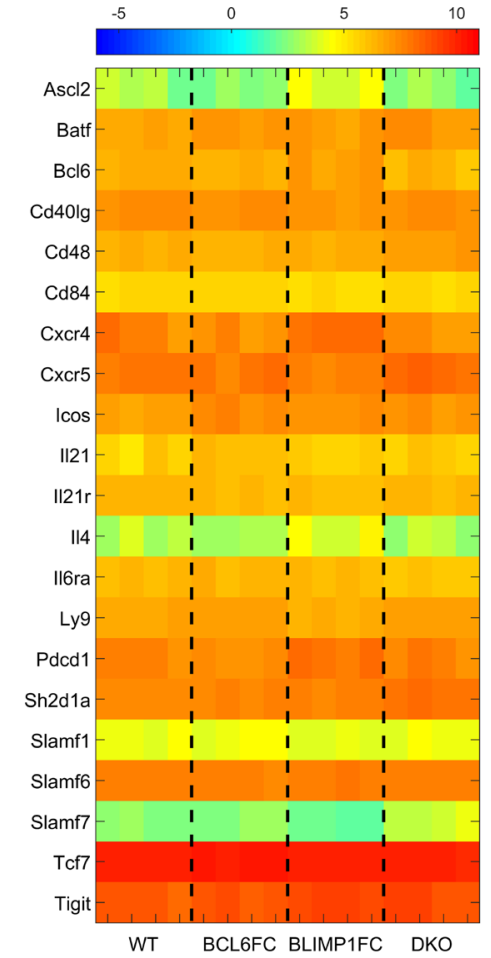

$\mathbf{F}$

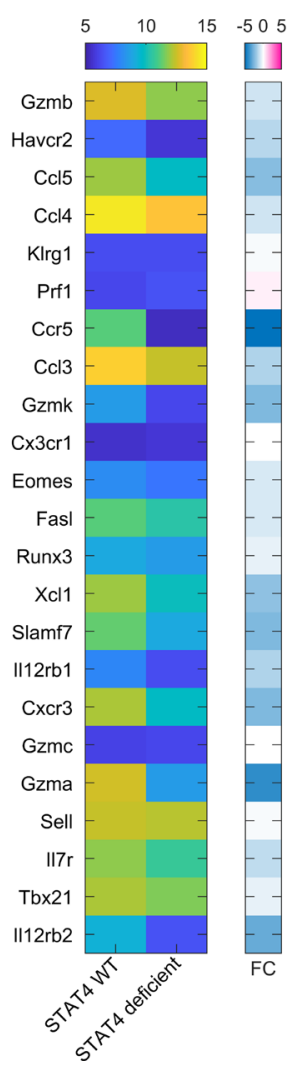


Figure 2. TFR cells repress a cytotoxic gene program in TFH cells. WT, Bcl6FC, Blimp1FC, and DKO mice were immunized twice with PCT ( $n=4$ ). Four weeks after the last immunization, TFH cells were isolated by FACS, RNA was then prepared and subjected to RNAseq. (A) Volcano plots showing differentially expressed genes (DEGs) for Bcl6FC versus WT (155 genes up, 410 genes down) and DKO versus Blimp1FC (517 genes up, 1041 down). Blue indicates downregulated genes and purple upregulated genes, using FDR $<0.05$ after multiple-test correction and fold change (FC) $<-1.8$ or $>1.8$ (linear). Gzmb is specifically marked in both plots. Gzmb is increased 9-fold in BcI6FC TFH cells compared with WT TFH cells, and Gzmb is increased 173-fold in DKO TFH cells compared with Blimp1FC TFH cells. (B) Heatmap showing expression assessed with RNAseq of 21 hallmark TFH genes sorted alphabetically. Color scale shows $\log _{2}$ RPKM of gene expression. (C) Fraction of genes associated with CD8 (left panel) or Stat4 (right panel) in all TFH expressed genes (gray bar), TFH DEGs upregulated for Bcl6FC (purple bar), or DKO (brown bar) versus WT. The CD8 or Stat4 genes were acquired from published data sets for DEGs in effector CD8 differentiation (18) and Stat4 regulation in Th1 cells (19). The $P$ values were calculated based on hypergeometric distribution, representing statistical significance of enrichment of CD8 and Stat4 genes in Bcl6FC or DKO upregulated DEGs compared with the occurrence in all expressed TFH genes. (D-F) A set of 23 genes was used to create heatmaps based on $\log _{2}$ RPKM gene expression for (D) WT, BcI6FC, Blimp1FC, and DKO TFH cells; (E) naive and effector CD8 ${ }^{+}$T cells (18); and (F) WT and Stat4 $4^{-1-}$ Th1 cells (19). In $\mathbf{E}$ and $\mathbf{F}, \mathrm{FCs}$ for the paired sets of genes are shown by a single-column heatmap. See Methods for statistical analysis of gene data sets.

wondered if cytotoxic TFH cells might develop in a natural context such as inflammation during virus infection. Using an RNAseq data set of TFH cells from simian immunodeficiency virus-infected (SIV-infected) macaques (23), we observed that there was a small but highly significant increase in expression of the $23 \mathrm{CD} 8$ effector cytotoxic genes in $\mathrm{SIV}^{+}$versus SIV- TFH cells (Figure 3C).

Cytotoxic gene expression in TFH cells and increased GCB apoptosis in TFR-deficient mice. To better characterize Gzmb-expressing cytotoxic-like TFH cells, we immunized WT, Bcl6FC, Blimp1FC, and DKO mice with sheep red blood cells (SRBCs), a model Ag that induces strong TFH responses. We stained TFH cells intracellularly for granzyme B protein by flow cytometry and saw a clearly distinct population of granzyme $\mathrm{B}^{+}$cells in DKO TFH cells that was not seen in the other types of TFH cells (Figure 4A). This finding was consistent with the higher level of CD8 effector gene upregulation in DKO TFH cells (Figure 2). We next examined Eomesodermin (Eomes), a protein highly expressed in $\mathrm{CD} 8^{+}$effector $\mathrm{T}$ cells and cytotoxic $\mathrm{CD} 4^{+} \mathrm{T}$ cells (24). DKO TFH cells also had a clear population of Eomes ${ }^{+} \mathrm{TFH}$ cells that was not observed in the other TFH cell types (Figure 4B). Costaining for both granzyme B and Eomes showed that all granzyme $\mathrm{B}^{+}$cells expressed Eomes, whereas there was a subset of Eomes ${ }^{+}$cells that did not express granzyme B (Figure $4 \mathrm{C}$ ). We also detected a well-defined population of DKO TFH cells expressing cell surface Klrg1 (Figure 5A). We therefore isolated $\mathrm{Klrg} 1^{+} \mathrm{TFH}$ cells by FACS to test gene expression compared to Klrg1- TFH cells (Figure 5B). Klrg1 ${ }^{+}$ DKO TFH cells had increased Gzmb mRNA and lower expression of the TFH genes Bcl6, Il4, and Il21, whereas Eomes, Ifng, and Prdm 1 were not significantly different between the two populations (Figure 5B). Klrg1 $1^{+} \mathrm{G}-$ $\mathrm{zmb}^{+}$DKO TFH cells likely have decreased helper potential due to lower Il4 and Il21, concurrent with a cytotoxic genotype. Similar to our results with PCT immunization, GC B cell responses were sharply lower in TFR-deficient mice after SRBC immunization (Figure 6, A-C). We then reasoned that cytotoxic TFH cells might kill GC B cells, leading to the losses of GC B cells seen in TFR-deficient mice. Analyzing cells directly ex vivo we observed a significant increase in apoptotic GC B cells in Bcl6FC and DKO mice compared with WT and Blimp1FC levels (Figure 6D). DKO GC B cells showed a 3-fold increase in apoptotic cells over Blimp1FC GC B cells, compared with a 2-fold increase in Bc16FC apoptotic GCB cells over WT GC B cells. This higher level of apoptosis is consistent with a higher level of cytotoxic TFH cells in DKO mice.

\section{Discussion}

In this study, we define a cytotoxic-like TFH cell subset that is repressed by TFR cells and is promoted by proinflammatory signals (virus infection, loss of Treg cell function). These cytotoxic-like TFH cells express high levels of granzyme B and Eomes proteins and express several other genes associated with CD8 ${ }^{+}$cytotoxic $\mathrm{T}$ cell function. Since the main function of TFH cells is to interact with B cells in the GC, cytotoxic-like TFH cells have the potential to kill B cells in the GC. Our findings therefore open up a possible new regulatory pathway for the GC and high-affinity Ab production. Additionally, TFR cells have been generally considered to repress the GC and Ab response (8-11). However, a recent study showed that TFR cells expressing IL-10 can act as helpers and promote Ag-specific IgG production and the GC response (12). Our data in this study show a potent helper effect of TFR cells on the GC that correlates with suppressed development of cytotoxic-like TFH cells. As shown in Supplemental Figure 2, injection of WT Tregs capable of developing into TFR cells into DKO mice repressed the abnormal production of cytotoxic-like TFH cells. Thus, TFR cells actively regulate the differentiation of TFH cells into different functional phenotypes. Furthermore, we have revealed what we believe is a new mechanism of TFR cell help in the GC response: inhibiting the development of cytotoxic-like TFH cells. 
A

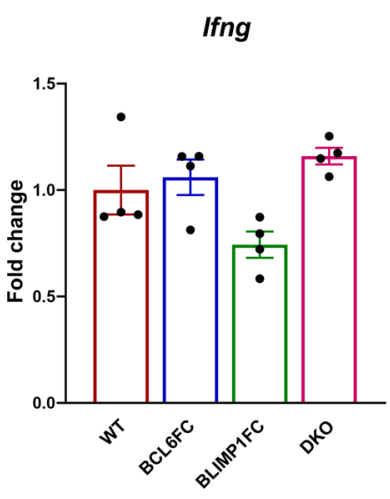

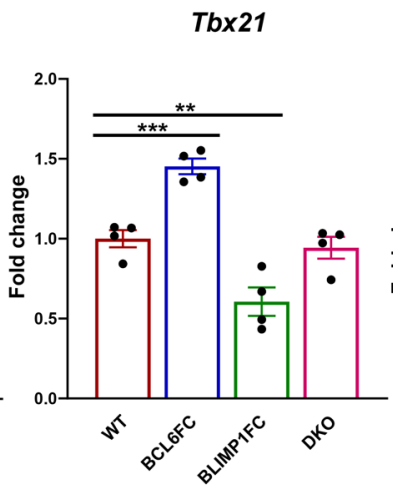

$T b \times 21$

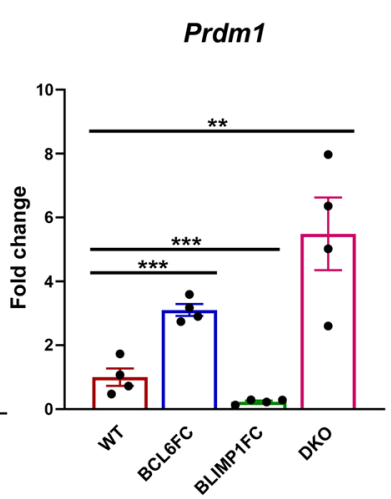

B

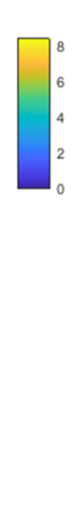

C

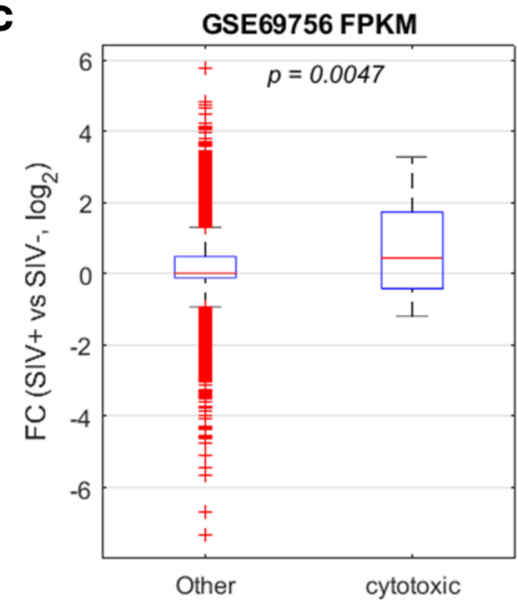

GSE105806 ThetRNASeq

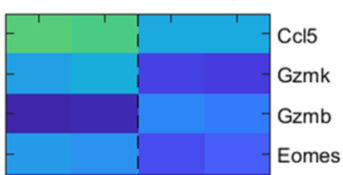

Ccl4

Ccr5

-Runx3

Ccl3

-Sell

-1112rb1

Prf1

Slamf7

Fasl

Havcr2

Gzma

Il12rb2

Gzmc

KIrg1

Cxcr3

Cx3cr 1

Tbx21 $-117 \mathrm{r}$

Tbet KO

Figure 3. Expression of Th1 genes in TFH cells, Tbet regulation of cytotoxic genes, and cytotoxic TFH genes upregulated after SIV infection. (A) Cene expression plots for Ifng, Tbx21 (Tbet), and Prdm1 (Blimp1) taken from the RNAseq data analyzed in Figure 2. WT (red) TFH cell average mRNA levels were set to 1 and fold changes are shown for Bcl6FC (blue), Blimp1FC (green), and DKO (magenta) TFH cells. The mRNA levels are taken from log 2 RPKM counts. $n=4$. ANOVA with Tukey's post hoc analysis was used to determine statistical significance. (B) Heatmap of the gene set of 23 CD8 ${ }^{+}$cytotoxic T cell genes described in Figure 2, D-F, versus published gene expression data for WT and Tbet-KO T cells (CSE105806). (C) Comparison of fold changes in genes from TFH cells from SIV+ infected macaques over uninfected TFH cells from uninfected macaques (published RNAseq data GSE69756). As a group, the 23 CD8 ${ }^{+}$ cytotoxic T cell genes described in Figure 2, D-F, show a statistically significant increase within the SIV+ TFH RNAseq data set over the SIV- TFH RNAseq data set ("cytotoxic" column), in comparison with all other noncytotoxic genes expressed in the TFH cells, which on average showed no statistically significant upregulation between the 2 types of TFH cells ("other" column). For statistical significance of overlap between 2 sets of genes, the $P$ value was calculated based on hypergeometric distribution.

Our analysis of TFH cells from SIV-infected macaques indicates that the cytotoxic-like TFH cell phenotype that develops in the absence of TFR cells can also develop in a normal (TFR cell sufficient) GC response after virus infection. Granzyme B-expressing circulating TFH cells have been found in humans, and the development of this population is Stat3 dependent (25). However, we were unable to find an enrichment of Stat 3 target genes in our TFH cell data (data not shown), suggesting there may be different origins for these circulating granzyme B-expressing TFH cells. Here, we saw a strong correlation of TFH cytotoxic genes with Stat 4 transcriptional function. Higher Stat 4 activity may indicate that IL-12 is increased in the GC environment in the absence of TFR cells. One key function of Tregs is to downmodulate dendritic cell function, including the secretion of cytokines such as IL-12 $(26,27)$. Like Tregs, TFR cells may function to suppress dendritic cells, but act in the GC and/or B cell follicle.

Very recently, granzyme B-expressing TFH cells have been found in GCs in human tonsillitis (28). How these human cytotoxic TFH cells develop is not clear, but they appear to be a defective or exhausted state of TFH cell differentiation induced by chronic infection (28). It is tempting to speculate that defective TFR cell function leads to the development of these human granzyme B-expressing TFH cells, but more research is required for answering this question. However, our results show that this cytotoxic TFH cell 
A
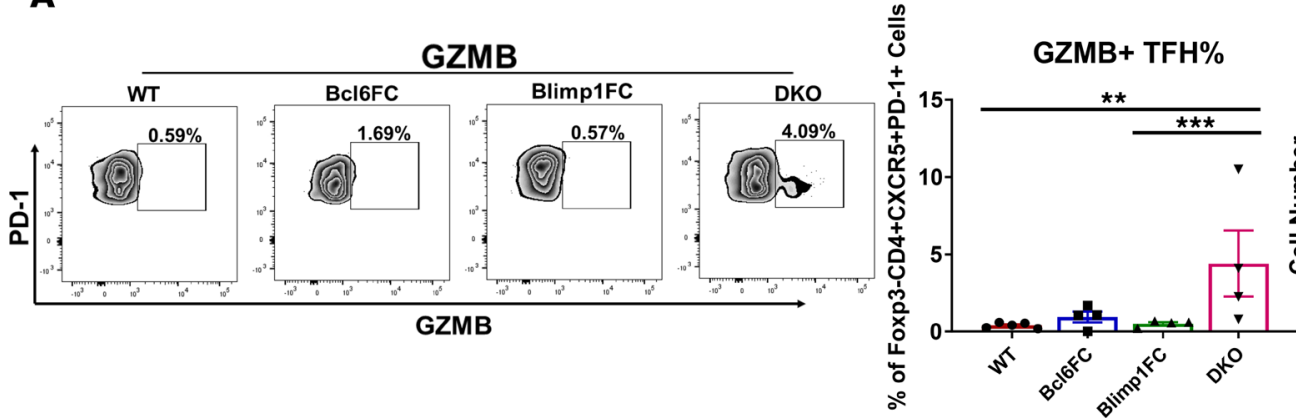

GZMB+ TFH\#

B
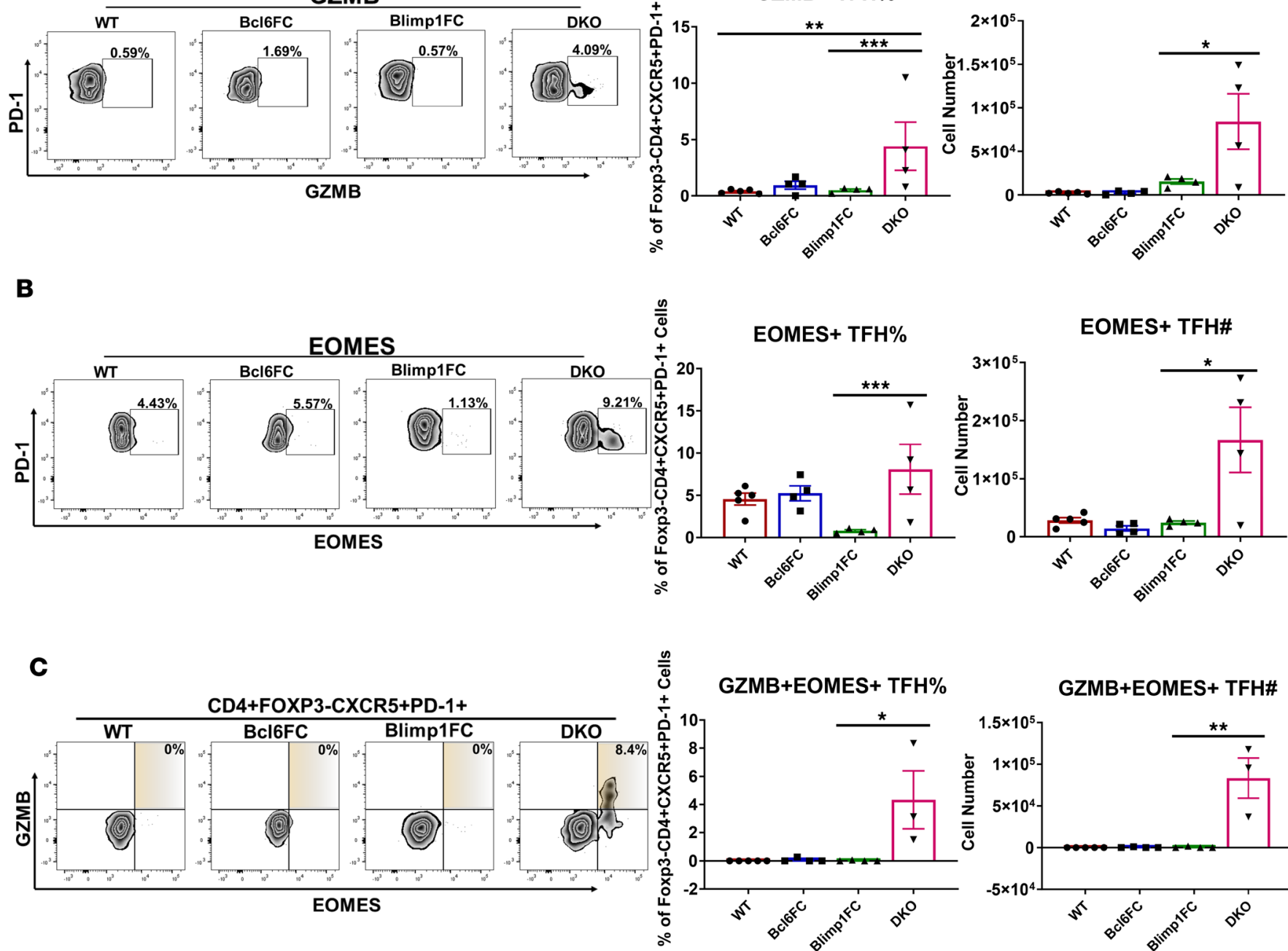

GZMB+EOMES+ TFH\#

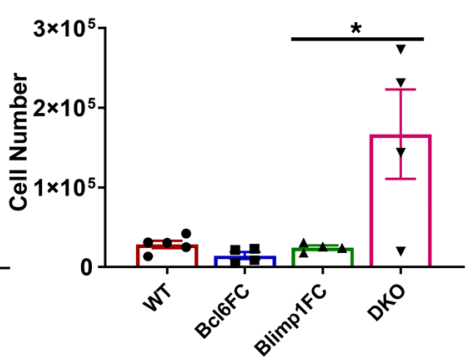

Figure 4. Cytotoxic gene expression in TFH cells and increased GC B cell apoptosis in TFR-deficient mice. Cytotoxic gene expression in TFH cells and increased GCB apoptosis in TFR-deficient mice. WT, BCI6FC, Blimp1FC, and DKO mice were immunized with SRBCs and 9 days later, spleen cells were analyzed by flow cytometry for CD4+FOXP3-PD-1+CXCR5+ TFH cells and staining with (A) anti-granzyme B (Czmb) Ab, (B) anti-Eomesodermin (Eomes) Ab, or (C) both Gzmb and Eomes Abs. Average Gzmb ${ }^{+}$(A), Eomes ${ }^{+}$(B), and Gzmb+Eomes ${ }^{+}$(C) TFH cells are quantitated and graphed as a percentage of TFH cells, and absolute number. A total of 100,000 cells were analyzed by flow cytometry and TFH cells $(0.5 \%-3 \%, 500-3,000)$ were gated for further analysis. $n=4$; experiment was repeated 3 times. ANOVA with Tukey's post hoc analysis was used to determine statistical significance.

pathway is conserved between mouse and humans and increases the possibility that TFR cells also control the development of human granzyme B-expressing TFH cells.

Overall, our findings raise the possibility that cytotoxic TFH cells are a normal part of the GC response and that granzyme $\mathrm{B}^{+} \mathrm{TFH}$ cells kill GC B cells. These cytotoxic-like TFH cells not only suppress the Ab response, as in the case of PCT immunization, but can fine-tune the types of IgG isotypes within the $\mathrm{Ab}$ response as shown with SRBC immunization (Supplemental Figure 3). Increased cytotoxic TFH cells in DKO mice correlated with a significant shift from in the ratio of IgG1 to IgG2b anti-SRBC Ab (Supplemental Figure 3). Cytotoxic TFH cells may also play a role in controlling virus infection in the GC, as we observed higher levels of cytotoxic genes in TFH cells after SIV infection. Augmenting the development of these aberrant TFH cells may be beneficial after virus infection. Cytotoxic CD4 ${ }^{+} \mathrm{T}$ cells have been studied previously by many investigators, but were never identified as a TFH cell subset (24). How TFR cells suppress granzyme B-expressing TFH cells and the precise function of granzyme B-expressing TFH cells will be important areas for future research. 
A

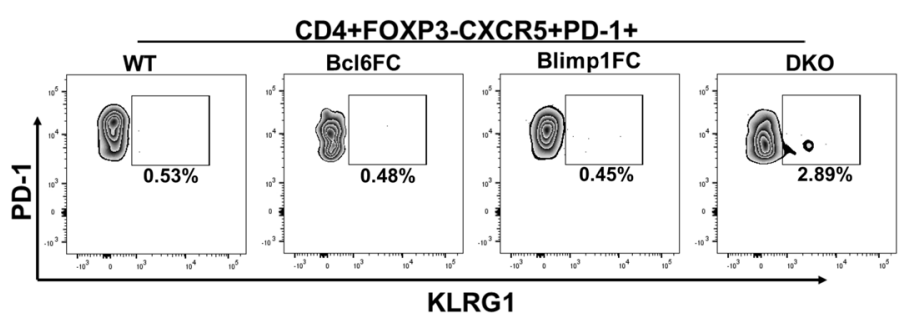

B

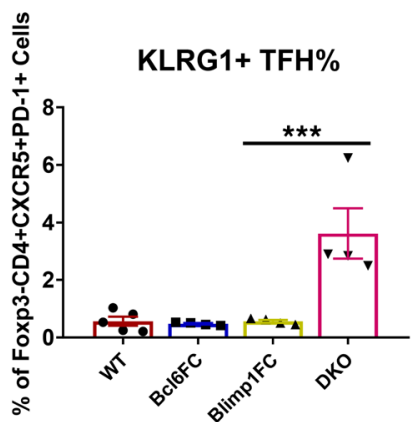

KLRG1+ TFH\#

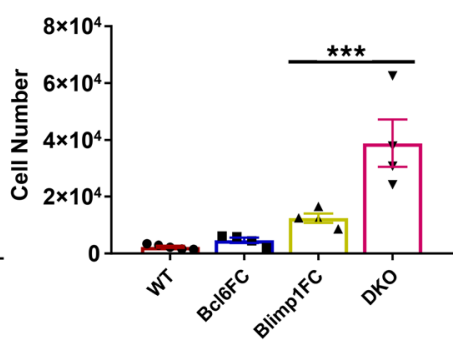

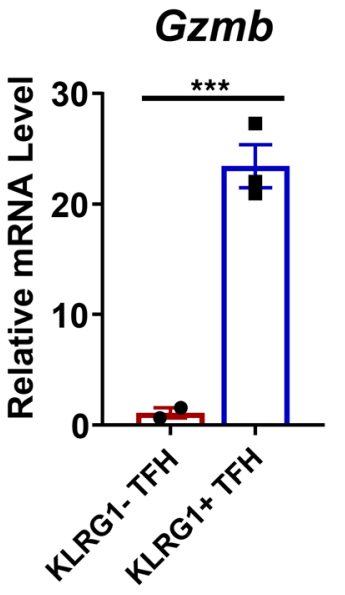

Bc/6

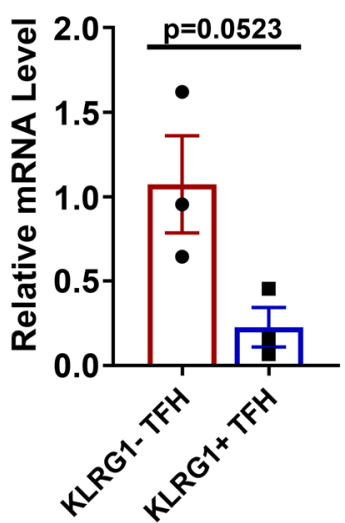

Eomes
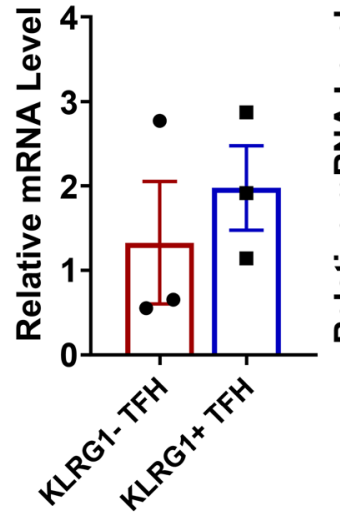

Prdm1

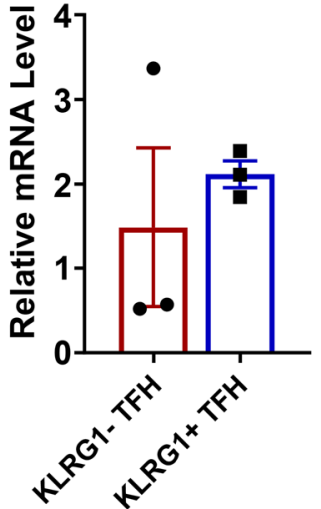

Ifng

114

$1 / 21$
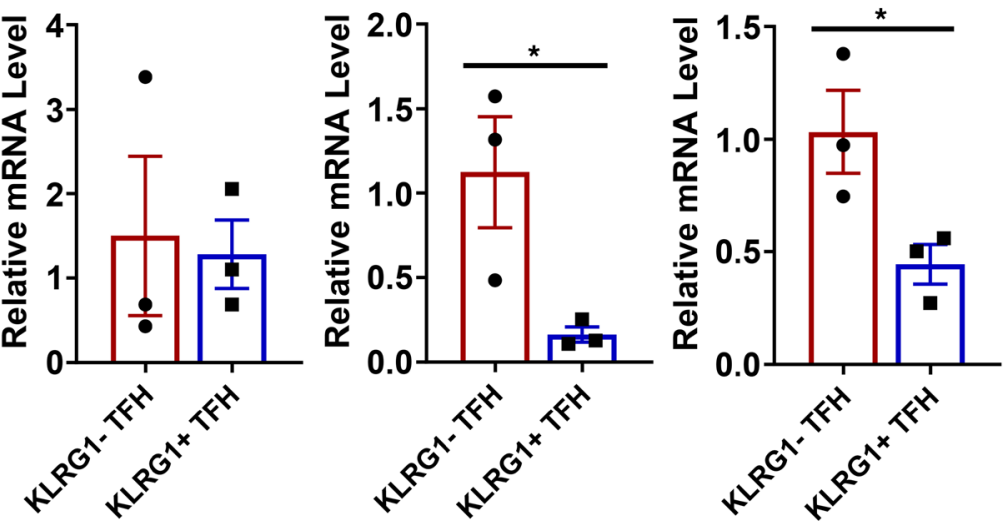

Figure 5. Cytotoxic gene expression is enriched in KIrg1+ TFH cells. WT, Bcl6FC, Blimp1FC, and DKO mice were immunized with SRBCs and spleens were analyzed for KIrg1 on TFH cell populations by flow cytometry. (A) Analysis of CD4 ${ }^{+}$FOXP3-PD-1+CXCR5 ${ }^{+}$TFH cells for KIrg1 expression 9 days after immunization. Average $\mathrm{KIrg} 1^{+} \mathrm{TFH}$ cells are quantitated and graphed as a percentage of TFH cells, and absolute number. $n=4$; experiment was repeated 3 times. Cells $(100,000$ total) were analyzed by flow cytometry and TFH cells $(0.5 \%-3 \%, 500-3,000)$ were gated for further analysis. ANOVA with Tukey's post hoc analysis was used to test for significant differences. (B) TFH cells from 4 DKO mice $(n=3)$ were separated into $\mathrm{Klrg}^{+}$and $\mathrm{KIrg}^{-}$populations by FACS, and RNA was prepared directly after sorting. RT-qPCR was used to analyze gene expression in $\mathrm{KIrg}^{+}$and $\mathrm{KIrg}^{-} \mathrm{TFH}$ cells. Graphs show relative expression for the indicated genes with the mRNA level in KIrg1- cells set to 1. This experiment was done twice with similar results. Approximately 15,000 KIrg1 ${ }^{+}$and approximately 500,000 KIrg1- TFH cells were sorted per mouse. Student's $t$ test was used to detect significant differences.

\section{Methods}

Mice. Foxp3-Yfp-cre only (WT) and Foxp3-Yfp-cre Bcl6-flox (Bcl6FC) mice were previously described (7, 29). Prdm1-flox mice (30) were crossed with Bcl6FC transgenic mice to generate Foxp3-Yfp-cre Prdm1-flox (Blimp1FC) and Foxp3-YFP-cre Prdm1-flox Bcl6-flox (DKO) mice. Male and female mice 6-10 weeks old were used. Mice were bred under specific pathogen-free conditions at the laboratory animal facility at Indiana University School of Medicine (IUSM).

Immunizations. On days 1 and 8, food was withdrawn from mice for 2 hours followed by gavage of $300 \mu \mathrm{L}$ per mouse with $1.5 \% \mathrm{NaHCO}_{3}$ in water i.g. One hour later, mice were orally immunized i.g. with $1 \mathrm{mg}$ per mouse of peanut extract (Greer Laboratories) together with $10 \mu \mathrm{g}$ per mouse of cholera toxin (Sigma-Aldrich) (15). For SRBC immunization, mice were injected i.p. with $1 \times 10^{9}$ SRBCs (Rockland Immunochemicals). Mice were sacrificed on indicated days and serum, mesenteric lymph nodes, and spleens were harvested. 
A

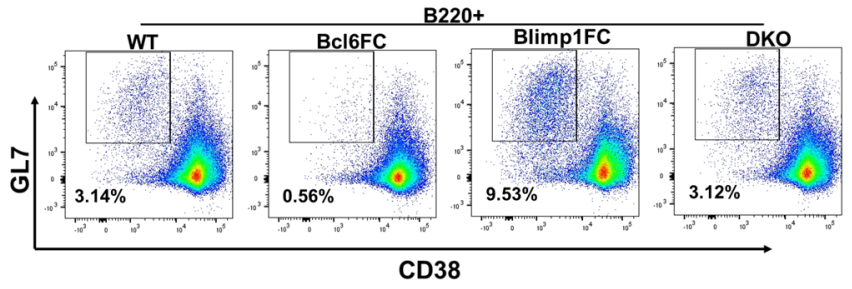

B
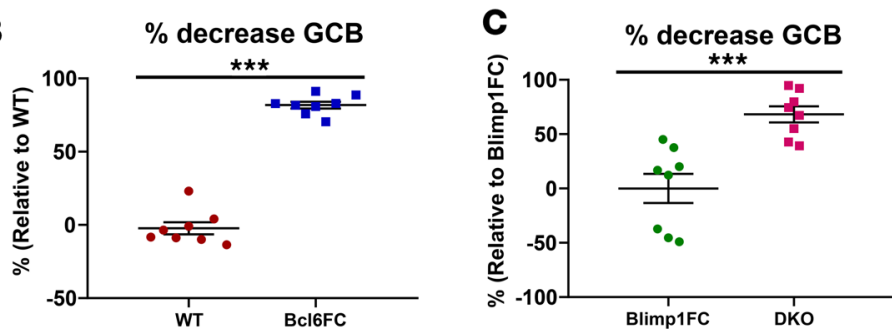

B220+GL7+CD38-

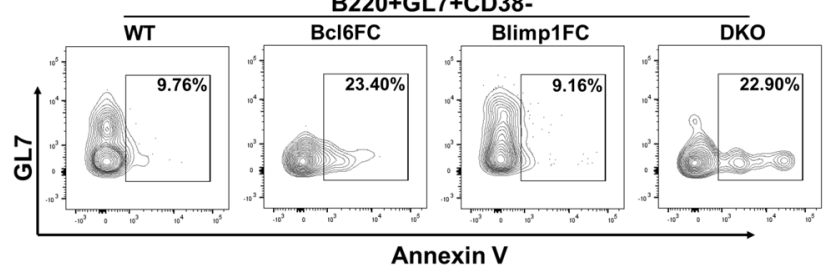

D

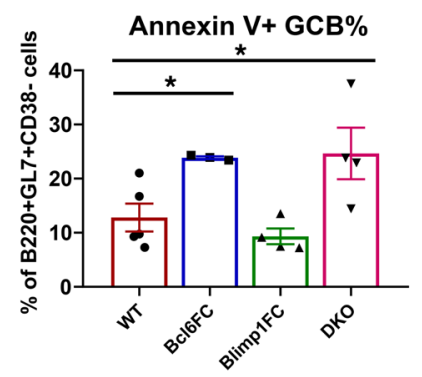

Figure 6. Increased GC B cell apoptosis in TFR-deficient mice. WT, Bcl6FC, Blimp1FC, and DKO mice were immunized with SRBCs and spleen cells were analyzed by flow cytometry for B220+CD38-CL7+ GC B cells. (A) Representative flow plots are shown. (B and C) The relative decrease of GC B cells in Bcl6FC and DKO mice is graphed with (C) BcI6FC mice compared with WT mice, and (D) DKO mice compared with Blimp1FC mice. CC B cell percentages were normalized to average control (WT in B, Blimp1FC in C) GC B cell percentages. Individual GC B cell gain or decrease was determined by the following formula: ([average control \% - normalized individual \%]/ average control $\%) \times 100$, with a positive value equaling loss and a negative value equaling gain. $n=8$; data in $\mathbf{B}$ and $\mathbf{C}$ are combined from 2 different experiments. Student's $t$ test was used to detect significant differences. (D) Annexin $\mathrm{V}^{+} \mathrm{CC}$ B cells in WT, Bcl6FC, Blimp1FC, and DKO mice, 3 days after immunization. Flow plots show GC B cells gated as in $\mathbf{A}$. Graphs show average annexin $V^{+}$GC $B$ cells. $n=4$; experiment was repeated 3 times; representative data are shown. ANOVA with Tukey's post hoc analysis was used to test for significant differences.

Flow cytometry. Cell suspensions were filtered through a $40-\mu \mathrm{m}$ cell strainer (Fisherbrand). Cells were washed and diluted in PBS with 1\% FBS and were stained with Fc block (BioXCell) for 5 minutes, followed by surface staining for the indicated markers. The following labeled Abs were used: anti-CXCR5 (L138D7), anti-PD-1 (29F.1A12), antiCD4 (RM4-5), anti-FOXP3 (MF-14), anti-CD38 (90), and anti-B220 (RA3-6B2) were obtained from BioLegend; antiGL7 (GL7) Ab was purchased from BD Pharmingen. Antiannexin $\mathrm{V} \mathrm{Ab}$ was obtained from BioLegend. For intracellular staining, after surface markers were stained, cells were fixed and stained with anti-granzyme B (GB11) and anti-Eomes (Dan11mag) using an eBioscience intracellular kit. All samples were acquired on an LSR2 flow cytometer (Becton Dickinson) and analyzed with FlowJo software.

$A b$ measurement. Titers of peanut-specific $\mathrm{Ab}$ in serum were measured by ELISA, as previously reported (31). For peanut-specific IgE, 96-well Nunc-Immuno plates (Sigma-Aldrich) were coated with IgE Ab (LO-ME-3, Bio-Rad) overnight at $4^{\circ} \mathrm{C}$. Wells were blocked with $1 \%$ BSA and diluted serum was added and incubated at room temperature for 2 hours. Peanut extract protein was labeled with biotin (Sigma-Aldrich) and added for 1 hour followed by adding poly-HRP streptavidin (Pierce Endogen) for 30 minutes.

Cell sorting, RNA isolation, and gene expression analysis. On day 36 after PCT challenge, CD4 ${ }^{+} \mathrm{T}$ cells were isolated from the spleen from mice using a CD4 T cell Macs isolation kit (Miltenyi Biotec). Cells were stained for CD4, CXCR5, and PD-1, and TFH cells were isolated by FACS. For isolation of Klrg1 ${ }^{+}$cells, mice were immunized with SRBC and TFH cells sorted as above but anti-Klrg1 Ab (2F1/KLRG1, BioLegend) was also used for staining. Total RNA was collected from freshly sorted cells using the RNeasy Plus Micro kit (QIAGEN). qPCR reactions were performed as previously reported (32).

RNAseq and bioinformatics analysis. RNAseq was performed by the IUSM Center for Molecular Genomics. KAPA mRNA HyperPrep methods were used for mRNA sequencing. Total RNA was evaluated for its quantity and quality using an Agilent Bioanalyzer 2100. For RNA quality, a RIN number of 7 or higher was used. Fifty nanograms of total RNA was used. cDNA library preparation included mRNA purification/enrichment, RNA fragmentation, cDNA synthesis, ligation of index adaptors, and amplification, following the KAPA mRNA Hyper Prep Kit Technical Data Sheet, KR1352 - v4.17 (Roche Corporate). Each resulting indexed library was quantified and quality accessed by Qubit and Agilent Bioanalyzer. Multiple libraries were pooled in equal molarity. Five microliters of 2-nM pooled libraries per lane were denatured, neutralized, and applied to the cBot for flow cell deposition and cluster amplification, before loading onto the HiSeq 4000 for 75-bp paired-end sequencing (Illumina, Inc.). Approximately 30 million reads per library were generated. A Phred quality score ( $\mathrm{Q}$ score) was used to measure the quality of sequencing. 
More than $90 \%$ of the sequencing reads reached Q30 (99.9\% base call accuracy). For sequence alignment and gene counts, the sequencing data were first assessed using FastQC (Babraham Bioinformatics) for quality control. Then all sequenced libraries were mapped to the mouse genome (mm10) using STAR RNAseq aligner with the parameter "--outSAMmapqUnique 60." The reads distribution across the genome was assessed using bamutils (from ngsutils). Uniquely mapped sequencing reads were assigned to mm10 refGene genes using featureCounts (from subread) with the parameters "-s 2 -p -Q 10." Quality control of sequencing and mapping results was summarized using MultiQC. Genes with read count per million (CPM) less than 0.5 in more than 4 of the samples were removed. The data were normalized using the trimmed mean of $\mathrm{M}$ values (TMM) method. Differential expression analysis was performed using edgeR. False discovery rate (FDR) was computed from $P$ values using the Benjamini-Hochberg procedure. DEGs were determined if their $P$ values were less than 0.05 after multiple-test correction with FDR adjustment and the amplitudes of fold changes (FCs) were larger than 1.8. RNAseq data were submitted to the NCBI Gene Expression Omnibus under accession number GSE133533.

Statistics. Flow cytometry and qPCR data analysis was done using GraphPad Prism software. Graph bars represent the mean \pm SEM. Unless otherwise stated, Student's $t$ test (2-tailed) or ANOVA with Tukey's post hoc analysis were used to determine significance. Significant differences $(P<0.05)$ are indicated in the figures. In order to examine the statistical significance of overlap between 2 sets of genes, we calculated the $P$ value based on hypergeometric distribution.

Study approval. Mice were handled according to protocols approved by the IUSM Institutional Animal Use and Care Committee (IACUC).

\section{Author contributions}

MMX helped design the study, performed all the biological experiments, analyzed the results, and prepared most of the figures. SF and JW performed bioinformatics analyses of the RNAseq data, integrated results with other published data sets, supported data interpretation, generated figures (Figure 2, Figure 3B, and Supplemental Figure 1), and wrote the methods part of the manuscript. QC performed some of the experiments. HL managed the mouse breeding and genotyping. ALD helped design the study, analyzed the results, and wrote the manuscript.

\section{Acknowledgments}

This work was supported by NIH R01 AI132771 to ALD. MMX was supported by a Careers in Immunology Fellowship from American Association of Immunologists. We thank Xiaoling Xuei and Yunlong Liu for their assistance with the RNAseq experiment. We thank Joseph Craft for helpful discussions and Mark Kaplan for critical reading of the manuscript.

Address correspondence to: Alexander Dent, Department of Microbiology and Immunology, 950 West Walnut Street, R2 302, Indiana University School of Medicine, Indianapolis, Indiana 46202, USA. Phone: 317.274.7524; Email: adent2@iupui.edu. Or to: Jun Wan, Department of Medical and Molecular Genetics, Collaborative Core for Cancer Bioinformatics (C3B), Indiana University School of Medicine, Indiana University School of Informatics and Computing, Indiana University-Purdue University Indianapolis, 410 West 10th Street, HITS 5013, Indianapolis, Indiana 46202, USA. Phone: 317.278.6445; Email: junwan@iu.edu.

1. Victora GD, Nussenzweig MC. Germinal centers. Annu Rev Immunol. 2012;30:429-457.

2. Fazilleau N, Mark L, McHeyzer-Williams LJ, McHeyzer-Williams MG. Follicular helper T cells: lineage and location. Immunity. 2009;30(3):324-335.

3. Crotty S. Follicular helper CD4 T cells (TFH). Annu Rev Immunol. 2011;29:621-663.

4. Linterman MA, et al. Foxp3 ${ }^{+}$follicular regulatory T cells control the germinal center response. Nat Med. 2011;17(8):975-982.

5. Chung Y, et al. Follicular regulatory T cells expressing Foxp3 and Bcl-6 suppress germinal center reactions. Nat Med. 2011;17(8):983-988

6. Wollenberg I, et al. Regulation of the germinal center reaction by Foxp3 $3^{+}$follicular regulatory T cells. J Immunol. 2011;187(9):4553-4560.

7. Wu H, et al. Follicular regulatory $\mathrm{T}$ cells repress cytokine production by follicular helper $\mathrm{T}$ cells and optimize IgG responses in mice. Eur J Immunol. 2016;46(5):1152-1161.

8. Sage PT, Sharpe AH. T follicular regulatory cells. Immunol Rev. 2016;271(1):246-259.

9. Xie MM, Dent AL. Unexpected help: follicular regulatory T cells in the germinal center. Front Immunol. 2018;9:1536.

10. Wing JB, Tekgüç M, Sakaguchi S. Control of germinal center responses by T-follicular regulatory cells. Front Immunol. 
2018;9:1910.

11. Fazilleau N, Aloulou M. Several follicular regulatory T cell subsets with distinct phenotype and function emerge during germinal center reactions. Front Immunol. 2018;9:1792.

12. Laidlaw BJ, et al. Interleukin-10 from $\mathrm{CD}^{+}$follicular regulatory $\mathrm{T}$ cells promotes the germinal center response. Sci Immunol. 2017;2(16):eaan4767.

13. Fu W, et al. Deficiency in T follicular regulatory cells promotes autoimmunity. J Exp Med. 2018;215(3):815-825.

14. Botta D, et al. Dynamic regulation of T follicular regulatory cell responses by interleukin 2 during influenza infection. Nat Immunol. 2017;18(11):1249-1260.

15. Li XM, et al. A murine model of peanut anaphylaxis: T- and B-cell responses to a major peanut allergen mimic human responses. J Allergy Clin Immunol. 2000;106(1 Pt 1):150-158.

16. Yang G, et al. Transcriptional repressor Blimp1 regulates follicular regulatory T-cell homeostasis and function. Immunology. 2018;153(1):105-117.

17. Cretney E, et al. The transcription factors Blimp-1 and IRF4 jointly control the differentiation and function of effector regulatory T cells. Nat Immunol. 2011;12(4):304-311.

18. Kim EH, et al. Signal integration by Akt regulates CD8 T cell effector and memory differentiation. J Immunol. 2012;188(9):4305-4314.

19. Wei L, et al. Discrete roles of STAT4 and STAT6 transcription factors in tuning epigenetic modifications and transcription during T helper cell differentiation. Immunity. 2010;32(6):840-851.

20. Herndler-Brandstetter D, et al. KLRG1 ${ }^{+}$Effector CD8 ${ }^{+} \mathrm{T}$ cells lose KLRG1, differentiate into all memory T cell lineages, and convey enhanced protective immunity. Immunity. 2018;48(4):716-729.e8.

21. Ogawa C, et al. Blimp-1 functions as a molecular switch to prevent inflammatory activity in Foxp ${ }^{+}$ROR $\gamma \mathrm{t}^{+}$regulatory $\mathrm{T}$ cells Cell Rep. 2018;25(1):19-28.e5.

22. Cretney E, et al. Characterization of Blimp-1 function in effector regulatory T cells. J Autoimmun. 2018;91:73-82.

23. Chowdhury A, et al. Decreased T follicular regulatory cell/T follicular helper cell (TFH) in simian immunodeficiency virus-infected rhesus macaques may contribute to accumulation of TFH in chronic infection. J Immunol. 2015;195(7):3237-3247.

24. Takeuchi A, Saito T. CD4 CTL, a cytotoxic subset of $\mathrm{CD}^{+} \mathrm{T}$ cells, their differentiation and function. Front Immunol. 2017;8:194.

25. Alshekaili J, et al. STAT3 regulates cytotoxicity of human $\mathrm{CD} 57^{+} \mathrm{CD} 4^{+} \mathrm{T}$ cells in blood and lymphoid follicles. Sci Rep. 2018;8(1):3529.

26. Larmonier N, et al. Tumor-derived CD4(+)CD25(+) regulatory T cell suppression of dendritic cell function involves TGF-beta and IL-10. Cancer Immunol Immunother. 2007;56(1):48-59.

27. Dercamp C, Chemin K, Caux C, Trinchieri G, Vicari AP. Distinct and overlapping roles of interleukin-10 and CD25 regulatory $\mathrm{T}$ cells in the inhibition of antitumor CD8 T-cell responses. Cancer Res. 2005;65(18):8479-8486.

28. Dan JM, et al. Recurrent group A Streptococcus tonsillitis is an immunosusceptibility disease involving antibody deficiency and aberrant $\mathrm{T}_{\mathrm{FH}}$ cells. Sci Transl Med. 2019;11(478):eaau3776.

29. Hollister $\mathrm{K}$, et al. Insights into the role of Bcl6 in follicular Th cells using a new conditional mutant mouse model. J Immunol. 2013;191(7):3705-3711.

30. Xie MM, et al. Bc16 promotes follicular helper T-cell differentiation and PD-1 expression in a Blimp1-independent manner in mice. Eur J Immunol. 2017;47(7):1136-1141.

31. Dolence JJ, et al. Airway exposure initiates peanut allergy by involving the IL-1 pathway and T follicular helper cells in mice. $J$ Allergy Clin Immunol. 2018;142(4):1144-1158.e8.

32. Xie MM, Amet T, Liu H, Yu Q, Dent AL. AMP kinase promotes Bcl6 expression in both mouse and human T cells. Mol Immunol. 2017;81:67-75. 